eISSN 2444-7986

DOI: https://doi.org/10.14201/orl201781.14992

\author{
Artículo original
}

\title{
EXPERIENCIA CON VIDEO HEAD IMPULSE TESTING (vHIT)
}

\section{Experience with Video Head Impulse Testing (vHIT)}

\author{
Carmen ÁLVAREZ-SANTACRUZ; María LÓPEZ-ROBLES; Diego HELLÍN-MESEGUER
}

Servicio de Otorrinolaringología. Hospital General Universitario Reina Sofía. Murcia. España.

Correspondencia: carmenasantacruz@gmail.com

Fecha de recepción: 13 de septiembre de 2016

Fecha de aceptación: 26 de septiembre de 2016

Fecha de publicación: 1 de octubre de 2016

Fecha de publicación del fascículo: 1 de marzo de 2017

Conflicto de intereses: Los autores declaran no tener conflictos de intereses

Imágenes: Los autores declaran haber obtenido las imágenes con el permiso de los pacientes

Política de derechos y autoarchivo: se permite el autoarchivo de la versión post-print (SHERPA/RoMEO)

Licencia CC BY-NC-ND. Licencia Creative Commons Atribución-NoComercial-SinDerivar 4.0 Internacional

(c) Universidad de Salamanca. Su comercialización está sujeta al permiso del editor

RESUMEN

Introducción y objetivo: El diagnóstico de la patología vestibular se basa, en la historia, la exploración y la prueba calórica. Esta última solo permite el estudio del canal semicircular horizontal y es mal tolerada. Como alternativa surge el vHIT (Video Head Impulse Test), que analiza todos los canales semicirculares y es menos molesta. El fundamento del siguiente estudio es reflejar nuestra experiencia y comparar con los métodos previos. Método: Realizamos un estudio observacional, prospectivo y descriptivo de un año de duración de todos los pacientes de nuestra Área de Salud que consultaron por clínica de mareo, inestabilidad o vértigo. Resultados: se estudiaron 155 pacientes que consultaron por mareo. Observamos un claro predominio del sexo femenino, siendo la Enfermedad de Meniére, la más frecuentemente diagnosticada. Se realizó la prueba calórica a los pacientes seleccionados para una mejor aproximación donde existía duda. Las relaciones estadísticas fueron significativas entre el sexo con la neuritis vestibular y la migraña vestibular. Igualmente, entre el reflejo vestíbuloocular y la prueba calórica, con las patologías anteriores. Discusión: El vHIT es técnica rápida y sencilla en su ejecución con la ventaja que es muy bien tolerada sin la producción de la sintomatología clásica de las pruebas calóricas. Sus disparidades en resultados se deben a las diferencias en las frecuencias estimuladas. Conclusiones: EI vHIT facilita el diagnóstico complementario de patología aguda siendo muy bien tolerado. Sin embargo, en patologías con compensación central, los resultados pueden ser normales, requiriendo la prueba calórica para una mejor aproximación diagnóstica.

PALABRAS CLAVE mareo; vértigo posicional paroxístico benigno; enfermedad de Ménière; neuritis vestibular; migraña vestibular

SUMMARY

Introduction and objective: The diagnosis and study of vestibular pathology has been always guided by the medical history, exploration and caloric test. The caloric test has some limitations because it only allows the study of horizontal semicircular canal and it is also poorly tolerated by patients. Alternatively, the VHIT (Video Head Impulse Test), allows the analysis of all semicircular channels being quicker to perform and less obtrusive. The objective of the following study is to reflect our initial experience with the VHIT and compare it with another diagnosis 
tests. Method: This is a observational, prospective and descriptive study, of one year of observation in our Healthcare center for all patients who described symptoms of dizziness, unsteadiness or vertigo. Results: A total of 155 patients were included. There was a clear predominance of females, being Meniere's disease the most frequently diagnosed entity. The diagnosis was reached by vHIT. Caloric test was also performed in patients without definite or doubt in the diagnosis. With the data, the statistical relationships were established, being significant between sex with vestibular neuritis and vestibular migraine. There was a statistically significant relationship between vestibule-ocular reflex and caloric test associated with the previous pathologies. Discussion: The head impulse assisted video is a quick, simple and well tolerated technique without adverse symptoms like the caloric test. The disparities of results are due to differences in the stimulated frequencies, being the vHIT more physiological. Conclusions: VHIT facilitates the complementary diagnosis of acute pathology, being a well-tolerated technique. However, in pathologies with central compensation the results by VHIT may be normal, requiring the caloric test for better diagnostic approach.

KEYWORDS

dizziness; benign paroxysmal positional vertigo; Ménière's disease; vestibular neuritis; vestibular migraine

\section{INTRODUCCIÓN}

El equilibrio resulta de la integración en el sistema nervioso central (SNC) de la información visual, propioceptiva y vestibular, lo que permite funciones tan importantes como el mantenimiento de la postura, la marcha y la orientación en el espacio. Cualquier perturbación de estos sistemas producirá una alteración de la estabilidad del individuo que puede manifestarse en forma de vértigo o inestabilidad [1]. El mantenimiento del equilibro requiere de al menos la indemnidad de dos de estos sistemas pues cada uno de forma individualizada ofrecen información incompleta. La información es procesada por el SNC mediante la comparación de modelos aprendidos, almacenados como patrones de referencia que dan lugar a una respuesta motora concreta y adecuada. De este modo, cuando las aferencias no encuentran el patrón correcto, tendrá lugar una resolución motora lenta o inadecuada.

Las respuestas elaboradas por el SNC se liberarán a través de la excitación o inhibición de varios sistemas:

- Vía espinal, cuya finalidad es mantener el tono, tanto en posición estática como en la marcha.

- Vía oculomotora, que permite el mantenimiento del enfoque en la fóvea de un objeto de interés, aunque la cabeza o el cuerpo se encuentren en movimiento.

- Vía neurovegetativa, que mediante conexiones con centros parasimpáticos presenta función reguladora de la presión arterial, frecuencia cardiaca, etc.

- Vía cortical, cuya finalidad es la de convertir el movimiento en sensación consciente.
Las áreas corticales que reciben las aferencias vestibulares en el hombre no se conocen totalmente.

- Vías vestíbulo-cerebelosas, que colaboran en el mantenimiento del equilibro y el ajuste del reflejo vestíbulo-ocular.

El sistema vestibular compuesto por un laberinto a cada lado, permite la elaboración espacial de un mapa tridimensional al que son referidas el resto de informaciones, permitiendo que las respuestas motoras sean adecuadamente proyectadas para el mantenimiento de la base de sustentación, tanto en reposo como en movimiento.

La estimulación de un laberinto, que será el del sentido del movimiento, conlleva la inhibición del contralateral por la acción de las conexiones vestíbulo-vestibulares, lo que se conoce como «predominio vestibular».

Las conexiones vestibuloespinales aumentarán el tono del lado de predominio y disminuirán el tono del opuesto, de forma que la musculatura espinal empuja hacia el lado contrario con la finalidad de mantener la postura.

Las vías vestibulooculares provocaran un desplazamiento lento de los ojos en sentido opuesto al movimiento, proporcionalmente al grado de excitación del laberinto contralateral. Finalmente, las conexiones vestibuloparasimpáticas producirán pequeños cambios vegetativos y las vestibulocorticales posibilitarán la percepción consciente del movimiento [2].

El vértigo es un síntoma de alarma consecuencia de una alteración de la orientación, percibida como un movimiento de nuestro cuerpo o el espacio que nos rodea y que puede tener 
carácter permanente, transitorio o recurrente. Es el síntoma característico de la patología del sistema vestibular, denominado periférico por ser secundario a una afectación de los receptores del oído interno. Etimológicamente deriva del griego vertere que significa girar, siendo la definición más ilustrativa la de «alucinación de movimiento». Es un síntoma frecuente en la población general, con una prevalencia del $5 \%$ y una incidencia de $1,4 \%$ anual. Su prevalencia aumenta con la edad, afectando hasta al $30 \%$ de los mayores de 60 años [3].

Las principales entidades que cursan con vértigo de origen periférico en la población general son, el vértigo posicional paroxístico benigno (VPPB), la migraña vestibular (MV), la neuritis Vestibular aguda (NV) y la enfermedad de Ménière (EM).

EI VÉRTIGO POSICIONAL PAROXÍSTICO BENIGNO SE estima que supone hasta el $80 \%$ de los casos de vértigo, clásicamente definido por episodios breves de giro de objetos asociados a cambios posturales y acompañados de cortejo vegetativo, no presentando ni hipoacusia ni acúfenos. Es un cuadro benigno, más frecuente entre la cuarta y sexta década de la vida, más prevalente en el sexo femenino. Su origen se encuentra en la salida de fragmentos de las otoconias utriculares hacia los canales semicirculares por los cambios posturales con la estimulación de las células sensoriales y, por tanto, con la aparición del nistagmo y la sintomatología característica.

La NEURITIS VESTIBULAR es la anulación súbita y generalmente unilateral de la función vestibular que da lugar a una crisis vertiginosa de más de 24 horas de duración y de inicio brusco, con un importante cortejo vegetativo y sin otra focalidad neurológica. Predomina en la tercera y cuarta década de la vida, sin diferencia entre sexos. Tiene un origen desconocido, aunque se han postulado varias etiologías, siendo las más aceptadas actualmente la lesión del nervio vestibular por virus herpes y las alteraciones vasculares que daría lugar a lesiones inflamatorias del mismo.

La American Academy of OtolaryngologyHead and Neck Surgery, define la ENFERMEDAD DE MÉNIĖRE como un síndrome idiopático de hidrops endolinfático definido clínicamente por episodios espontáneos de vértigo recurrente, hipoacusia neurosensorial fluctuante, plenitud ótica y acúfenos. La etiología es desconocida, así como la incidencia y evoluciona hacia un deterioro progresivo de la función laberíntica y auditiva [4].

La MIGRAÑA VESTIBULAR es la causa más frecuente de vértigo espontáneo recurrente. Afecta aproximadamente al $10 \%$ de los pacientes migrañosos, puede aparecer a cualquier edad y afecta predominantemente a mujeres. Además, puede existir una asociación familiar que habla de un posible origen genético de la enfermedad. Suele manifestarse como una crisis de vértigo espontáneo o posicional cuya duración puede ser de segundos hasta días. El vértigo puede anteceder a la cefalea, acontecer juntos, o aparecer después de ella. La pérdida de audición o el acúfeno no son síntomas frecuentes, sin embargo, la fotofobia, la fonofobia o el aura visual sí son acompañantes habituales [5].

El diagnóstico de estas patologías sigue basándose en la historia clínica y la exploración otoneurológica completa que incluya la naturaleza del desequilibrio, desencadenantes, duración de los síntomas, asociación de síntomas neurológicos, cocleares o cardiovasculares, trastornos médicos generales concomitantes, traumatismos recientes y fármacos [6].

Las pruebas complementarias se sustentan en la información obtenida del análisis de los reflejos vestibulooculares (RVO) y vestibuloespinales, ya que no es posible el estudio directo de la función vestibular en sí misma.

Estas pruebas tienen su vigente histórico en la prueba calórica realizada por primera vez por Bárány en 1906 y hasta nuestros días, se ha considerado como la prueba complementaria más útil en la exploración vestibular. Se basa en el estudio del reflejo vestibuloocular, ya definido con anterioridad, como la respuesta provocada por el estímulo vestibular durante los movimientos de la cabeza que se traduce en un movimiento ocular con un mínimo retraso compensador en dirección contraria y misma velocidad. Técnicamente consiste en instilar aire o agua en el conducto auditivo externo para producir una estimulación térmica del canal semicircular horizontal que da lugar a una respuesta refleja vestibular, manifestada como nistagmo que puede registrarse para su valoración. Esta prueba permite el estudio únicamente del canal semicircular horizontal, siendo además una prueba mal tolerada al tratarse de 
un estímulo no fisiológico y desencadenar un intenso reflejo nauseoso [7].

Sin embargo, más recientemente, la exploración clínica de cada uno de los receptores puede ser individualizada con la prueba del impulso cefálico, sistematizada por Halmagyi y Curthoys. La valoración que se realiza es la de la posición ocular, es decir, donde se encuentra el ojo al finalizar el impulso cuando le pedimos al paciente que mantenga la mirada fija en un punto o si aparecen sacadas durante el mismo. Es una prueba subjetiva pero muy útil, ya que en la crisis aguda de vértigo la respuesta normal es el factor predictivo más importante ante la sospecha de un infarto cerebeloso.

La exploración instrumental en la que se basa este impulso ya se realizaba de forma muy detallada con la Bobina Corneal en Campo Magnético, sin embargo, es una prueba poco práctica en el entorno clínico habitual y menos aún en situaciones de urgencia por su carácter molesto para el paciente, de difícil de interpretación y larga duración en la realización [8].

Como alternativa a esta técnica surge recientemente la prueba del impulso cefálico con registro de vídeo o Video Head Impulse Test ( $v H I T)$, basado en la medición del reflejo vestibuloocular a velocidades fisiológicas. Consiste en un registro gráfico de la maniobra impulsiva descrita por Halmagyi y Curthoys, tratándose de una prueba rápida, poco molesta y relativamente sencilla de realizar que nos permite el estudio de los 6 canales semicirculares [9].

Por lo anterior, el principal objetivo de este estudio es valorar nuestra primera experiencia con este nuevo método de apoyo diagnóstico en las principales patologías que cursan con vértigo de origen periférico.

Como objetivos secundarios, buscaremos conocer los datos epidemiológicos de la patología vestibular distribuida por género y edad en nuestra área de salud, la utilidad y eficacia de la técnica en los diferentes diagnósticos vestibulares, comparar, en casos seleccionados, con respecto a la prueba calórica y determinar si la prueba calórica puede ser sustituida por la prueba objeto del estudio, así como, valorar la tolerancia de la prueba con respecto a los métodos de exploración aplicadas con anterioridad.

\section{MATERIAL Y MÉTODO}

Se estudiaron 155 pacientes pertenecientes al Área VII de Salud de la Región de Murcia que consultaron por clínica vertiginosa en el periodo del 1 de marzo de 2015 al 29 de febrero de 2016. Al 100\% de los sujetos se les realizó un vHIT y a 52 pacientes se les completó la exploración con una prueba calórica para una mejor aproximación diagnóstica.

Los pacientes a estudio son aquellos que acudieron a consultas externas de otorrinolaringología con clínica de mareo, vértigo o inestabilidad entre el mes y los tres meses anteriores a la realización de la misma.

Cada resultado obtenido se dividió en normal o patológico, realizando en primer lugar un estudio para clasificar los hallazgos obtenidos.

Se realizó una búsqueda bibliográfica en Scielo, Pubmed, Uptodate y Google Académico. Se seleccionaron aquellos archivos tanto nacionales como extranjeros que informan sobre los diferentes hallazgos encontrados mediante esta técnica en referencia a la localización de canales afectados según las diferentes patologías a estudio, frecuencias de estimulación aplicadas y comparación en caso de ser posible con la prueba calórica, así como la tolerancia de la misma para comparar los resultados obtenidos y los publicados hasta el momento.

Como criterios de exclusión se tomaron las alteraciones de la motilidad ocular o pérdida importante de la agudeza visual del ojo derecho, las alteraciones severas de la columna cervical y los portadores de cualquier dispositivo o prótesis que dificulte o artefacte el registro.

Se les realizó un estudio inicial con una exploración clínica vestibular sistemática completa, cuantificando el nistagmo tanto espontáneo como tras la realización de la maniobra de DixHallpike, bajo visualización directa. En función de la clínica, los pacientes fueron sometidos a prueba calórica y/o audiometría quedando igualmente reflejados los resultados en el estudio.

El reflejo vestibuloocular se exploró clínicamente acorde a las indicaciones de Halmagyi, sin equipo adicional, realizando tres impulsos a cada lado de forma aleatoria, considerándose normal la ausencia de sacadas al finalizar el movimiento.

Una vez realizada la exploración clínica, los pacientes seleccionados se sometieron a la exploración videoasistida del RVO. Para ello,

(c) Ediciones Universidad de Salamanca / CC BY-NC-ND 
se utilizó un equipo de vHIT (video Head-Impulse Testing, GN Optometrics, Dinamarca) formado por unas gafas que constan de una cámara, un giroscopio, una cinta elástica regulable y un proyector láser, conectados a un software de registro, situado en el ojo derecho.

Para la realización del test, el sujeto a estudio fue colocado cómodamente sentado a un metro de distancia de un punto concreto. La posición adecuada de las gafas resultó fundamental con la finalidad de garantizar una estabilidad suficiente durante los movimientos cefálicos que imprimimos sobre el paciente. Para ello, las gafas se colocaron en su posición habitual con una adecuada fijación de las mismas mediante la cinta elástica, asegurando que la cámara situada en el ojo derecho no se viera interferida con las pestañas del paciente ni se desplazase al realizar los giros de cabeza.

La calibración del sistema se hizo de acuerdo a las normas de Optometrics, por medio de dos puntos láser que se apagan y encienden de forma cambiante, indicando al paciente su seguimiento con la mirada sin movimientos cefálicos, asegurando el mantenimiento de la cabeza del sujeto en posición recta.

Para la realización de la prueba y con el paciente mirando fijamente al punto equidistante previamente definido, se imprimieron 20 impulsos rápidos (media de 150 a $400 \%$ s) alternantes a derecha e izquierda en el plano horizontal según la técnica descrita por Halmagyi y Curthoys, con la finalidad de valorar los canales semicirculares laterales.

Una vez realizados, se colocó al paciente en la misma posición, pero con la cabeza levemente girada a 30 grados hacia la derecha en el plano horizontal y se realizaron 10 movimientos alternantes hacia arriba y abajo en el plano vertical. Posteriormente se realizó el mismo movimiento, pero con la cabeza hacia la izquierda. Estos movimientos tienen la finalidad de estudiar los canales semicirculares situados en los planos sinérgicos.

Las variables recogidas para el estudio fueron: número de historia clínica, sexo, edad, clínica previa, realización de prueba calórica y su resultado, resultados del v-HIT: alteración de los canales semicirculares, alteración de la ganancia, sospecha diagnóstica previa y sintomatología otológica (hipoacusia y acúfenos).
La respuesta definitiva se obtuvo valorando dos aspectos de los impulsos realizados en los diferentes planos del espacio: la ganancia del reflejo y las sacadas de refijación. Los valores de las ganancias considerados normales son los cercanos a la unidad, siendo aproximadamente de 0,94 en los canales horizontales por ello, se consideró un valor de ganancia patológico aquel por debajo de 0,8 y 0,7 , respectivamente. Las sacadas de refijación se dividieron en covert o encubiertas que son las que tienen lugar durante el impulso y en evidentes u overt, cuando ha finalizado el mismo.

La información se analizó utilizando el paquete estadístico PASW Statistics 20 para Windows (SPSS Inc., Chicago, IL, USA). Se llevó a cabo un análisis de la normalidad de los datos referentes a la edad de los pacientes previo a su análisis estadístico mediante el test de Kolmogorov-Smirnov con un nivel de significancia de $p<0,05$. Además, por tablas de contingencia, utilizando el estadístico Chi-cuadrado con un nivel de significancia de $p<0,05$ se estudiaron las relaciones entre los posibles resultados obtenidos.

\section{RESULTADOS}

De los 155 pacientes el 63,87\% (99 casos) fueron mujeres y el $36,1 \%$ (56 casos) fueron hombres con edades comprendidas entre 11 y 83 años con una media de edad de $52 \pm 18$ años (media \pm desviación típica).

Respecto a la prueba vHIT, 84 pacientes $(54,2 \%)$ presentaron una alteración en la ganancia del RVO. Según la clínica y los resultados obtenidos en las pruebas diagnósticas aplicadas, los pacientes se distribuyeron por enfermedades (Tabla 1).

El vértigo posicional paroxístico benigno se diagnosticó en un $11 \%$ de los casos, apareciendo un $23,5 \%$ en hombres y un $76,4 \%$ en mujeres, con edades comprendidas entre los 39 y los 82 años y una media de edad de $59 \pm$ 12 años. Los episodios previos fueron referidos en un $76,5 \%$ de los pacientes. Del total, el $52,9 \%$ presentó hipoacusia neurosensorial y un $11,8 \%$ acúfenos no asociados a pérdida auditiva. El 41,2\% tuvieron alteración de la ganancia del RVO. El canal semicircular anterior (CSA) derecho se presentó afectado en un $29,4 \%$, en un $23,5 \%$ para CSA izquierdo, en un $17,6 \%$ para el canal semicircular posterior
Rev. ORL, 2017, 8, 1, pp. 5-15 
(CSP) derecho, en un 11,8\% para el canal semicircular lateral (CSL) izquierdo y en un $9 \%$ tanto para CSP izquierdo como para CSL derecho.

Tabla 1. Distribución de pacientes diagnosticados de vértigo posicional paroxístico benigno (VPPB), enfermedad de Ménière (EM), migraña vestibular (MV) y neuritis vestibular (NV).

\begin{tabular}{|l|c|c|}
\hline Patología & N. $^{\circ}$ de casos & \% del total \\
\hline VPPB & 17 & 11 \\
\hline EM & 25 & 16,1 \\
\hline MV & 20 & 12,9 \\
\hline NV & 22 & 14,2 \\
\hline Otras patologías & 71 & 45,8 \\
\hline Total & 155 & 100 \\
\hline
\end{tabular}

La enfermedad de Ménière se presentó en un $16,1 \%$ de los casos siendo predominante en mujeres $(72 \%)$. Las edades estuvieron comprendidas entre 12 y 72 años con una media de edad de $52 \pm 15$. El $96 \%$ de los casos refirieron episodios previos y se observó que la afectación del oído izquierdo presentó una ligera predominancia $(54,4 \%)$ con respecto al derecho. El $92 \%$ de los pacientes presentaron hipoacusia neurosensorial y un $48 \%$ de los casos presentó acúfenos. El $32 \%$ de los pacientes presentaron alteración de la ganancia detectada por el vHIT, siendo los canales por orden de afectación: en igualdad de aparición CSA derecho, CSA izquierdo, CSL izquierdo y CSP derecho con un 16\%, seguido del CSP izquierdo con un $12 \%$ y CSL derecho con un $8 \%$.

La migraña vestibular fue diagnosticada en un $12,9 \%$ de los pacientes de los cuales el $90 \%$ refirieron episodios previos. Los pacientes afectados fueron predominantemente mujeres $(90 \%)$ con edades entre 12 y 73 años con una edad media de $43 \pm 17$ años. La prueba del vHIT mostró una alteración de RVO en un 15\% de los casos afectando al CSA derecho, CSA izquierdo y CSP izquierdo con la misma frecuencia (10\%), ambos canales laterales aparecieron afectados en igual porcentaje (5\%), no apareciendo afectación del CSP derecho en ningún caso. La hipoacusia neurosensorial se presentó en un $20 \%$ casos y el $25 \%$ presentaron asociación a acúfenos, de los cuales, sólo en 2 casos se asoció a hipoacusia.

La neuritis vestibular se presentó en un 14,2\%, en pacientes con edades comprendidas entre los 24 y 80 años, con una media de edad de $52 \pm 16$ años y predominio en mujeres $(68,2 \%)$. La afectación del oído izquierdo fue más frecuente, apareciendo en un $55,6 \%$ de los casos. El $40,9 \%$ de los casos refirió clínica previa al último episodio y un $59,1 \%$ de estos pacientes presentaron alteraciones de la ganancia, siendo los canales predominantemente afectados los posteriores (CSP derecho en un $40 \%$ y CSP izquierdo en un 27,3\%). El 63,6\% presentó hipoacusia neurosensorial y el $36,4 \%$ refirió presencia de acúfenos durante el episodio.

En función a la sintomatología y la sospecha diagnóstica, los pacientes fueron programados para la realización de videonistagmografía. Se le realizó a un total de 52 pacientes, de los cuales el $53,73 \%$ obtuvo un resultado positivo para disfunción vestibular según esta técnica, considerándose patológicos los valores superiores al $20 \%$.

Se realizaron pruebas calóricas a 4 pacientes con sospecha diagnóstica de VPPB. La edad media de estos pacientes fue de $65 \pm 6$ años y un $75 \%$ fueron mujeres. El total de las pruebas calóricas obtuvieron un resultado negativo, al igual que la prueba del impulso cefálico que no mostró alteración de la ganancia en ninguno de los canales semicirculares (Tabla 2).

En 15 pacientes con sospecha de Enfermedad de Ménière se realizó la técnica calórica. La edad media de estos pacientes fue de $50 \pm 14$ años y el $73,3 \%$ del total fueron mujeres. Un $75,4 \%$ de estas pruebas fueron positivas para déficit vestibular mientras que la disminución de la ganancia del RVO solo apareció en un $33,3 \%$ de los casos. El canal más afectado fue el CSA izquierdo con un $26,7 \%$ en esta muestra. El CSL derecho no aparece afectado en ninguno de los casos. La lateralidad fue levemente más acentuada hacia el oído izquierdo (Tabla 2).

Se realizó prueba calórica a 6 pacientes con sospecha de migraña vestibular con una edad media de $45 \pm 11$ años, siendo un $66,7 \%$ de los casos mujeres. La prueba calórica fue positiva en un $50 \%$ de los casos, mientras que la alteración de la ganancia medida por vHIT solo apareció en un $16,7 \%$ de los casos. Para esta 
entidad, solo se vieron afectados los canales CSA izquierdo, CSA derecho, CSL derecho y CSP izquierdo (Tabla 2).

Se realizaron pruebas calóricas a 15 pacientes con diagnóstico o sospecha de neuritis vestibular aguda con media de edad de 56,36 \pm 14,19 años y el $71,4 \%$ fueron hombres. El85,6\% de las pruebas calóricas resultaron positivas para disfunción vestibular pero la alteración de la ganancia medida por VHIT apareció alterada en el $50 \%$ de los casos. El canal más afectado fue el CSP derecho que aparece con resultado alterado en un $42,9 \%$, seguido por el CSA derecho en un $28,6 \%$. El canal que con menos frecuencia aparece alterado es el CSL izquierdo, los restantes canales tienen semejantes porcentajes de afectación en esta patología (Tabla 2).

Con los datos obtenidos se realizó el Test de la Chi-cuadrado de Pearson para analizar si existía relación estadísticamente significativa entre el diagnóstico realizado y distintos parámetros evaluados.

No se obtuvo relación estadísticamente significativa entre los diagnósticos y el sexo de los pacientes, excepto en el caso de la migraña vestibular y la hipofunción vestibular donde si apreció una relación significativa $(p<0,05)$, siendo más frecuentes en mujeres y en hombres respectivamente (Tabla 3 ).

Tabla 2. Distribución de pacientes con alteración del reflejo vestibuloocular (en porcentaje). Resultado positivo para prueba calórica y canales afectados.

\begin{tabular}{|c|c|c|c|c|c|c|c|c|}
\hline $\begin{array}{l}\frac{\pi}{0} \\
\frac{0}{0} \\
\frac{0}{0} \\
0\end{array}$ & 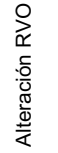 & 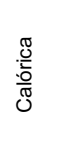 & 怘 & ভ্ & $\begin{array}{l}\stackrel{0}{0} \\
\text { ஸु }\end{array}$ & 吕 & $\begin{array}{l}0 \\
0 \\
\text { D } \\
\text { D }\end{array}$ & $\begin{array}{l}0 \\
\text { के } \\
0\end{array}$ \\
\hline $\begin{array}{l}\text { VP } \\
\text { PB }\end{array}$ & 0 & 0 & 0 & 0 & 0 & 0 & 0 & 0 \\
\hline EM & 33,3 & 60 & 13,3 & 26,7 & $0 \%$ & 13,3 & 20 & 20 \\
\hline MV & 16,7 & 50 & 16,7 & 16,7 & 16,7 & 0 & 0 & 16,7 \\
\hline HV & 50 & 78,6 & 28,6 & 21,4 & 21,4 & 14,3 & 42,9 & 21,4 \\
\hline
\end{tabular}
(canal semicircular posterior), D (derecho), I (izquierdo), VPP (vértigo posicional paroxístico), EM (enfermedad de Ménière), MV (migraña vestibular), NV (neuritis vestibular), RVO (reflejo vestibuloocular).

En cuanto a los resultados de las pruebas diagnósticas realizadas (calóricas y vHIT) se obtuvieron relaciones estadísticamente significativas en la alteración de la ganancia del RVO con respecto a la migraña vestibular, $(p<0,043)$ y tanto de la prueba calórica como de alteración de la ganancia en la neuritis vestibular $(p<0,011)$. Para el resto, no existe una relación entre el diagnóstico y un resultado positivo de estas pruebas (Tabla 4).

Tabla 3. Nivel de significación del test Chi-cuadrado aplicado a los datos de diagnóstico clínico y sexo de los pacientes.

\begin{tabular}{|l|c|}
\hline & Sexo de los pacientes \\
\hline $\begin{array}{l}\text { Vértigo Posicional Paroxís- } \\
\text { tico Benigno }\end{array}$ & 0,252 \\
\hline Enfermedad de Ménière & 0,356 \\
\hline Migraña Vestibular & $0,009^{*}$ \\
\hline Neuritis Vestibular & $0,001^{*}$ \\
\hline
\end{tabular}

El test Chi Cuadrado refleja además que no existe predominancia de oído afecto ni en la Enfermedad de Ménière ni en el caso de hipofunción vestibular. Tampoco se observó relación estadísticamente significativa entre los canales afectados y las diferentes patologías.

Tabla 4. Nivel de significación del test Chi-cuadrado aplicado a los datos de diagnóstico clínico y resultados alteración de RVO (reflejo vestibuloocular) y prueba calórica.

\begin{tabular}{|l|c|c|}
\hline & Calórica & Alteración RVO \\
\hline $\begin{array}{l}\text { Vértigo Posicional } \\
\text { Paroxístico Benigno }\end{array}$ & 0,331 & 0,577 \\
\hline $\begin{array}{l}\text { Enfermedad de Mé- } \\
\text { nière }\end{array}$ & 0,565 & 0,843 \\
\hline Migraña Vestibular & 0,847 & $0,043^{*}$ \\
\hline Neuritis Vestibular & $0,030^{*}$ & $0,011^{*}$ \\
\hline
\end{tabular}

\section{DISCUSIÓN}

Los resultados obtenidos en nuestro trabajo permiten afirmar que el estudio del RVO mediante VHIT es un procedimiento que aporta información diferente y complementaria al estudio convencional.

La prueba calórica utilizada tradicionalmente requiere por parte del explorador una curva de 
aprendizaje prolongada que le dote de una adecuada experiencia, tanto en la realización como en la interpretación de resultados. A nivel de la práctica clínica diaria, la ejecución de la prueba calórica requiere un tiempo de aproximadamente una hora, junto con la producción al paciente de una intensa sensación vertiginosa con la consiguiente estimulación vegetativa, al instilar aire o agua a diferentes temperaturas en el oído, lo que hace que sea bastante desagradable y rechazada.

El vHIT es un nuevo sistema diagnóstico que surge con la finalidad, entre otras, de reducir los tiempos y la sintomatología producida por la prueba anterior. Por nuestra experiencia, el aprendizaje resulta relativamente sencillo y la consecución de una adecuada ejecución e interpretación se adquiere de forma rápida.

Al igual que en la literatura, presenta un alto índice de reproductibilidad junto con ausencia de la sintomatología típica que acompaña a la prueba calórica y por tanto, una mejor tolerancia. En nuestro estudio, ninguno de nuestros pacientes refirió ningún efecto secundario relacionado [10].

Esto resulta de especial interés en población pediátrica, donde se está observando un aumento de la patología vestibular en los últimos años y, donde las pruebas de rutina son también muy mal toleradas con un importante aumento de la ansiedad. El vHIT presenta una alta eficiencia y reproductibilidad en pacientes con edades comprendidas entre los 3 y 16 años frente a otras técnicas [11, 12], igual que observamos nosotros, aunque con edades comprendidas entre los 11 y los 16 años.

La exploración video asistida es, desde el punto de vista económico y de optimización de recursos, un método que puede conseguir diagnósticos mucho más rápidos, con menor estrés y un ahorro en el tiempo de exploración de hasta 10 minutos por paciente [13].

Por otra parte, y según los estudios consultados, los resultados no se ven modificados en función de la edad únicamente más allá de los 90 años, apreciándose un pequeño decremento de la velocidad en los canales posteriores, lo que permite la aplicación de los mismos criterios a todos los grupos de edad [14, 15]. En líneas generales, presenta una baja sensibilidad asociada a una alta especificidad, teniendo altos valores predictivos positivos si existe una alta sospecha clínica por parte de otorrinolaringólogo.

Si se compara la información ofrecida por la maniobra de impulso cefálico video asistido y los resultados de la prueba calórica, la sensibilidad de la prueba oscila entre el 34 y $39 \%$ con una especificidad de 97 a 100\%. Aplicado a la clínica, en aquellos pacientes con déficits vestibulares grandes es altamente improbable que la prueba sea negativa y, de esta forma, si consideramos solamente déficits vestibulares muy grandes, la sensibilidad de la prueba aumenta [16], lo que tiene especial importancia en los diagnósticos rápidos durante el proceso agudo de la enfermedad, en la primera atención, que suele ser de carácter urgente.

Por otra parte, no incluida en el repertorio de pruebas empleadas en nuestro medio, se encuentran la «Bobina de Búsqueda Escleral en Campo Magnético»-Scheral-Search-Coils-. Se trata de dos bobinas metálicas que se colocan sobre la superficie escleral y que permiten una adecuada medida del RVO, al igual que el método descrito. Este sistema supone un aumento de la sensibilidad y especificidad frente a las pruebas clásicas de detección de la patología vestibular; sin embargo, se trata de una prueba invasiva, cara, muy molesta para el paciente y de difícil implementación [17].

Pese a su sensibilidad y especificidad, los análisis comparativos demuestran que la prueba del impulso cefálico videoasistido es equivalente a la «Bobina de Búsqueda Escleral en Campo Magnético» en la identificación de déficit vestibulares periféricos, con la diferencia de que es mucho más sencilla de utilizar y mejor tolerada por los pacientes [18].

La técnica registra los movimientos cefálicos junto con los movimientos compensatorios oculares, facilitando el análisis de las características del estímulo-respuesta del RVO a distintos grados de magnitud y correlacionándolos con los distintos grados de respuesta, convirtiendo a esta prueba en una herramienta objetiva en la evaluación del reflejo vestíbulo ocular. Por otra parte, permite una mejor valoración de la localización de la lesión, al evaluar la función dinámica de todos los canales semicirculares a diferencia de la prueba calórica que se limita a los canales laterales. Sin embargo, por lo comentado con anterioridad sus 
resultados deben ser interpretados en combinación con los obtenidos en resto de exploraciones, ya que la interpretación de los mismos de forma aislada puede conducir a errores [19, 20].

Una diferencia apreciada la constituyen las frecuencias estimuladas, donde la prueba calórica realiza una estimulación con frecuencias muy bajas que no son las fisiológicas o las aplicadas en la vida cotidiana, y el vHIT reproduce la estimulación a frecuencias superiores, más fisiológicas. Esto podría explicar por qué los pacientes de la población estudiada con ambas pruebas y catalogados como Enfermedad de Ménière, migraña vestibular o hipofunción vestibular, tienen mayor porcentaje de resultados positivos cuando se compara la técnica calórica frente al impulso cefálico videoasistido [21].

Si bien el VHIT es una prueba que permite una mejor optimización del tiempo, esto no es cierto en los casos de enfermedad de Ménière en fase crónica o compensada previamente y no diagnosticada recomendándose en ellos, la prueba calórica como primera técnica de estudio [22].

A pesar su baja sensibilidad, ningún método de evaluación de la función vestibular ha demostrado tener mayor sensibilidad que el resto de pruebas empleadas en otoneurología, por lo que el examen aislado con una de ellas de forma exclusiva ofrece resultados poco confiables.

Los estudios realizados sobre este tema revelan la importancia de una buena anamnesis, que ha demostrado una capacidad predictiva del vértigo postural paroxístico benigno de $90 \%$, en la enfermedad de Ménière del $86 \%$, y en la neuronitis vestibular del $63 \%$, otorgándole un valor predictivo general de $84 \%$. Es por ello que la adecuada historia clínica y exploración física es fundamental tanto a la hora de seleccionar a los pacientes a los que se les va a realizar pruebas vestibulares para llegar a un diagnóstico definitivo [23].

Consecuencia de lo anterior, la buena exploración clínica y tipificación de los pacientes conllevó a que la enfermedad de Ménière, así como la hipofunción vestibular, fueran las entidades estudiadas con más frecuencia en nuestra población, ya que el VPPB, a pesar de ser el más frecuente, tiene un diagnóstico y un tratamiento clínico, basado en maniobras posicionales y que en muy pocas ocasiones precisa de pruebas complementaria.

No obstante, llama la atención los casos estudiados de VPPB que presentaron alteraciones de la ganancia. Los canales semicirculares más afectados fueron los anteriores, no coincidiendo con el cuadro fisiopatológico clásico, que suele afectar a los canales posteriores. Solo el $17 \%$ presentó alteración de este canal, lo que nos puede orientar hacia una exploración deficiente o bien a un falso positivo. Sin embargo, a aquellos pacientes estudiados con la prueba vestibular calórica y vHIT, no se apreció alteración vestibular en ninguna de las técnicas realizadas. La afectación de un solo canal fue más frecuente que la afectación de dos o más [24].

Como se ha comentado anteriormente, la enfermedad de Ménière es la enfermedad más frecuentemente estudiada en nuestro trabajo. Los pacientes diagnosticados solo presentaron alteración de la ganancia en un $32 \%$, mientras que los resultados por prueba calórica positiva alcanzaron el $75,4 \%$. Este hecho lo atribuimos a la estimulación de frecuencias diferentes y a la compensación central. Por este motivo pensamos que la exploración de RVO video asistido puede arrojar un resultado normal en estos pacientes. La lateralidad, en cuanto al oído afectado, no muestra relación estadísticamente significativa.

La migraña vestibular se presenta en mujeres jóvenes, con relación estadísticamente significativa $(p<0,05)$. En el $15 \%$ de estas migrañas se observa una alteración de la ganancia en la exploración vestibular video asistida. Cuando se compara con la prueba calórica, en el $50 \%$ se observa un resultado positivo. La migraña vestibular es un diagnóstico de exclusión y se asocia a resultados positivos en las pruebas calóricas en el $60-70 \%$ de los casos en periodo intercrítico, de forma muy parecida a lo que ocurre con la Enfermedad de Ménière. En nuestra serie, al tratarse de un estudio con una muestra más limitada y de corta duración, algunos factores externos podrían confundir resultados al presentar estas dos entidades síntomas similares [25].

La neuritis vestibular es más frecuente en varones, con relación estadísticamente significativa; sin embargo, la afectación de un oído u otro es indistinta. El reflejo vestíbulo ocular 
aparece alterado en el $60 \%$ mientras que la prueba calórica es positiva en casi el $85,6 \%$ de los casos.

Por lo descrito, podemos afirmar que la prueba del impulso cefálico permite un diagnóstico rápido y muy bien tolerado de los déficits vestibulares agudos, así como su posterior evolución con seguimiento. No lo es tanto para alteraciones crónicas no diagnosticadas, donde la prueba calórica sigue aportando información muy valiosa. No obstante, esta nueva técnica, se está aplicando en el diagnóstico diferencial urgente de la patología de origen central, gracias a su versatilidad, lo que abre un amplio abanico de aplicaciones para este método diagnóstico [26, 27].

\section{CONCLUSIONES}

La clínica de mareo tiene mayor prevalencia en mujeres y en edades medias de la vida, aunque no existan diferencias estadísticamente significativas. En la distribución por patologías, aunque sin significación estadística, el sexo predominante fue el femenino salvo en la migraña vestibular, con la que sí hay relación estadísticamente significativa. También existe asociación estadísticamente significativa en la hipofunción vestibular con el sexo masculino. El vHIT es una técnica sencilla de realizar permitiendo diagnósticos rápidos en la patología aguda vestibular. Sus resultados no son interpretables en el vértigo posicional paroxístico benigno, donde por definición no existe lesión $\mathrm{y}$, por tanto, sus resultados no son valorables. La historia clínica en este caso, así como la exploración, es el método de elección para su diagnóstico y el vHIT no parece aportar más datos. En la enfermedad de Ménière o en la hipofunción vestibular en fase de intercrisis, el vHIT es menos preciso a la hora de poder establecer un primer diagnóstico de estas patologías. Por ello, concluimos que el vHIT es válido en un diagnóstico inicial rápido, pero su normalidad debe orientar hacia la realización de la prueba calórica si se considera clínicamente necesaria.Encontramos diferencias estadísticamente significativas en la medición del reflejo vestibuloocular y la migraña vestibular y de éste último junto con la prueba calórica en la neuritis vestibular. El resto de diagnósticos no presentaron asociación, al igual que la afectación de un determinado canal o la predominancia de un oído respecto al otro en ninguna de las patologías estudiadas. Todos nuestros pacientes han tolerado la prueba y ninguno ha referido incidencias durante o posterior a su realización.

Aunque aún nos quedan estudios por realizar, podemos concluir que la prueba de impulso cefálico video-asistido debería ser la primera prueba complementaria en los pacientes que consultan por clínica de mareo. En función a los datos recogidos, tanto en la historia y la exploración, así como el vHIT, realizaríamos la prueba calórica para obtener más datos en caso de precisarlo. Por los datos obtenidos en nuestro estudio, podemos concluir que el vHIT no sustituye a la prueba calórica en determinadas patologías, pero por su tolerancia, fácil y rápida realización, es una prueba muy útil en el área de la otoneurología.

\section{BIBLIOGRAFÍA}

1. Becker W, Heinz Naumann H, Rudolf Pfaltz C. Otorrinolaringología, Manual ilustrado. Barcelona: Doyma; 1992.

2. Suárez C, Gil-Carcedo LM, Medina JE, Marco J, Ortega P, Trinidad J. Tratado de Otorrinolaringología y Cirugía de Cabeza $Y$ Cuello. Tomo II: Madrid; Panamericana. 2008.

3. Murdin L, Schilder AG. Epidemiology of balance symptoms and disorders in the community: a systematic review. Otol Neurotol. 2015;36(3):387-92.

4. Bisdorff AR, Staab JP, Newman-Toker DE. Overview of the International Classification of Vestibular Disorders. Neurol Clin. 2015;33(3):541-50.

5. Batuecas Caletrío A, Martín Sanz E, Trinidad Ruiz G, Espinosa Sánchez JM, Alemán López $O$. Migraña vestibular: Diagnóstico y tratamiento. Rev Soc Otorrinolaringol Castilla Leon Cantab La Rioja. 2013; 4(5):21-9. Disponible en: http://hdl.handle.net/10366/124501. [Citado el 30-09-2016].

6. Alemán-López $\mathrm{O}$, Pérez-Garríguez H, PérezVásquez P, Arán-González I, Martin-Sanz E. Survey on the state of otoneurology in Spain. Acta Otorrinolaringol Esp. 2015;66(6):30915.

7. Furman J, JS Barton J, Evaluation of the patient with vertigo. UpToDate. 2014. Disponible en: www.uptodate.com. [Citado el 0812- 2014]. 
8. Pérez-Fernández N, Gallegos-Constantino V, Barona Lleo L, Manrique Huarte R. Exploración Clínica y videoasistida del reflejo vestíbulo-oculomotor: análisis comparativo. Acta Otorrinolaringol. 2012;63(6): 429-35.

9. Carriel C, Rojas M. Prueba de impulso cefálico: Bases fisiológicas y métodos de registro del reflejo vestíbulo oculomotor. Rev Otorrinolaringol Cir Cabeza Cuello. 2013;73(2):206-12.

10. Breinbauer H, Anabalón JL. Prueba de Impulso Cefálico. Rev Otorrinolaringol Cir Cabeza Cuello 2011;71:123-30.

11. Hülse R, Hörmann $K$, Servais JJ, Hülse $M$, Wenzel A. Clinical experience with video Head Impulse Test in children. Int J Pediatr Otorhinolaryngol. 2015;79(8):1288-93.

12. Hamilton SS, Zhou G, Brodsky JR. Video head impulse testing (VHIT) in the pediatric population. Int $\mathrm{J}$ Pediatr Otorhinolaryngol. 2015;79(8):1283-7.

13. Rambold Holger A. Economic management of vertigo/dizziness disease in a county hospital: video-head-impulse test vs. caloric irrigation. Eur Arch Otorhinolaryngol. 2015;272(10):2621-8.

14. Yang CJ, Lee JY, Kang BC, Lee HS, Yoo $\mathrm{MH}$, Park HJ. Quantitative analysis of gains and catch-up saccades of video-head-impulse testing by age in normal subjects. Clin Otolaryngol. 2016;41(5):532-8.

15. Maheu M, Houde MS, Landry SP, Champoux F. The Effects of Aging on Clinical Vestibular Evaluations. Front Neurol. 2015; 22;6:205.

16. Batuecas Caletrío A, Muñoz Herrera A, Bronstein AM. Importancia de la maniobra de impulso óculo-cefálico o Head impulse test en la consulta otorrinolaringológica general. Rev Soc Oorrinolaringol Castilla Leon Cantab La Rioja. 2012; 3 (32): 266-70. Disponible en: http://hdl.handle.net/10366/124477. [Citado el 30-09-2016].

17. MacDougall H.G, Weber KP, McGarvie LA, Halmagyi GM., Curthoys IS. The video head impulse test. Diagnostic accuracy in peripheral vestibulopathy. Ann Neurol. 2009;73(14):1134-41.
18. Magliulo G, lannella G, Gagliardi S, Massimo R. A 1-year follow-up study with C-VEMPs, O-VEMPs and video head impulse testing in vestibular neuritis. Eur Arch Otorhinolaryngol. 2014; 272(11):3277-81.

19. Carriel C, Rojas M. Prueba de impulso cefálico: Bases fisiológicas y métodos de registro del reflejo vestíbulo oculomotor. Rev Otorrinolaringol. Cir. Cabeza Cuello 2013;73(2):206-12.

20. MacDougall HG, McGarvie LA, Halmagyi GM, Curthoys IS, Weber KP. Application of the video head impulse test to detect vertical semicircular canal dysfunction. Otol Neurotol. 2013;34(6):974-9.

21. Bell SL, Barker $F$, Heselton $H$, MacKenzie $E$, Dewhurst D, Sanderson A. A study of the relationship between the video head impulse test and air calorics. Eur Arch Otorhinolaryngol. 2015;272(5):1287-94.

22. Rambold Holger A. Economic management of vertigo/dizziness disease in a county hospital: video-head-impulse test vs. caloric irrigation. Eur Arch Otorhinolaryngol. 2015;272(10):2621-8.

23. Peña A. El examen vestibular abreviado, descripción, interpretación y análisis. Rev Otorrinolaringol Cir Cabeza Cuello 2011;71: 13544.

24. Miłoński J, Pietkiewicz $P$, Bielińska $M$, Kuśmierczyk K, Olszewski J. The use of videonystagmography head impulse test (VHIT) in the diagnostics of semicircular canal injuries in patients with vertigo. Int $\mathrm{J}$ Occup Med Environ Health. 2014;27(4):583-90.

25. Yoo MH, Kim SH, Lee JY, Yang CJ, Lee HS, Park HJ. Results of video head impulse and caloric tests in 36 patients with vestibular migraine and 23 patients with vestibular neuritis: A preliminary report. Clin Otolaryngol. 2015; 9. doi: 10.1111/coa.12556.

26. Weber KP, MacDougall HG, Halmagyi GM, Curthoys IS. Impulsive testing of semicircularcanal function using video-oculography. Ann NY Acad Sci. 2009;1164:486-91.

27. Newman-Toker DE, Saber Tehrani AS, Mantokoudis G, Pula JH, Guede $\mathrm{Cl}$, Kerber KA, Blitz A, Ying SH, Hsieh YH, Rothman RE, Hanley DF, Zee DS, Kattah JC. Quantitative video-oculography to help diagnose stroke in acute vertigo and dizziness: toward an ECG for the eyes. Stroke. 2013;44(4):1158-61. 